\title{
Predicting the presence of coronary artery disease by transesophageal echocardiography
}

\author{
Matthias Schneider (D) Houtan Heidari · Hong Ran · Christian Roth - Christian Hengstenberg - Thomas Binder • \\ Georg Goliasch
}

Received: 20 November 2019 / Accepted: 21 May 2020 / Published online: 12 June 2020

(C) The Author(s) 2020

\begin{abstract}
Summary
Background The accuracy of ultrasound signs as predictors for the presence of coronary artery disease (CAD) has been evaluated extensively in the 1990s and 2000s. Imaging quality has improved tremendously over the last decades.

Hypothesis High-end ultrasound systems allow for accurate prediction of the presence or absence of CAD. Methods All patients who underwent a transesophageal echocardiography examination (TEE) between 2007 and 2016 and who had coronary angiography within 24 months before or after the TEE were retrospectively evaluated.

Results A total of 242 patients fulfilled the inclusion criteria, $60 \%$ were male. Mean age was 70 years ( $\mathrm{SD} \pm 13$ years). In multivariate regression analysis, plaque in the ascending aorta (odds ratio [OR] 2.51, 95\% confidence interval $[\mathrm{CI}] 1.18-5.32, p=0.017$ ), plaque in at least one of the thoracic aortic segments (OR 2.07, 95\% CI 1.02-4.22, $p=0.045$ ), and the presence of mitral annular calcification (MAC, OR 1.84, 95\% CI 1.01-3.36, $p=0.046$ ) were predictors of significant CAD. The isolated finding of aortic stenosis (AS) (OR 2.53, 95\%CI 1.23-5.21, $p=0.012$ ) was a significant predictor for the absence of normal coronary arteries. Conclusion With an negative predictive value (NPV) of $80 \%$, the absence of MAC, AS, and aortic plaque makes the presence of significant CAD unlikely. If at
\end{abstract}

M. Schneider, MD $(\bowtie) \cdot$ H. Heidari, MD $\cdot$ C. Roth, MD . C. Hengstenberg, MD · T. Binder, MD · G. Goliasch, MD, PhD Department of Internal Medicine II, Medical University of Vienna, Waehringer Guertel 18-20, 1090 Vienna, Austria matthias.schneider@meduniwien.ac.at

\section{H. Ran, MD}

Department of Echocardiography, Nanjing First Hospital Affiliated to Nanjing Medical University, Nanjing, China least mild AS is present, normal coronary arteries are improbable.

Keywords TEE $\cdot$ Aorta $\cdot$ Aortic plaque $\cdot$ Mitral annular calcification $\cdot$ Aortic stenosis

\section{Introduction}

The accuracy of ultrasound signs as predictors for the presence of coronary artery disease (CAD) has been evaluated extensively in the 1990s and early 2000s. Imaging quality has changed tremendously since. The data used for earlier analyses were based on ultrasound images, which do not compare to today's imaging systems, some of the earlier studies on mitral annular calcification (MAC) being based on M-mode findings [1-3].

Of the patients with peripheral arterial disease $41 \%$ have concomitant coronary or cerebrovascular disease [4]. In the Framingham study the presence of MAC predicted all cause death, cardiovascular death, and cardiovascular disease [5]. The presence of MAC was an independent predictor of cardiovascular events in another study, the risk being directly related to severity of MAC [6]. Premature MAC and aortic valve sclerosis (AVS) in young patients were found to be a predictor of reduced myocardial perfusion [7] and for the presence of CAD [8-10]. Exposure to multiple cardiovascular risk factors at a young age is associated with increased prevalence of MAC and AVS later in life [11]. Mitral annular calcification and AS detected in TTE predict the presence of significant CAD [12]. The presence of both MAC and aortic plaques detected in TEE, was associated with a higher incidence of stroke and cardiac death in a patient group investigated in the 1990s [13]. Others could show the association of inflammation and presence of MAC [14] as well as an association between the 
presence of MAC with AVS, aortic plaque, and CAD $[15,16]$; however, in a recent study, the clinical significance of MAC was challenged and it was discussed to be a mere age-related degenerative finding rather than a predictor of cardiovascular disease [17].

The absence of atherosclerosis in the thoracic aorta resulted in a negative predictive value of $90-99 \%$ for the presence of significant CAD in a number of previous studies [18-21]. Gu et al. showed that the presence of aortic plaques, especially those in the descending aorta, are associated with the presence of significant CAD [22]. The presence of severe aortic plaque in TEE is a predictor for poor survival as well as a risk for ischemic stroke [23, 24].

The aim of this study was to re-evaluate the predictive accuracy of MAC, AVS, and aortic plaque detected in TEE examination on high-end ultrasound machines for the diagnosis of CAD.

\section{Methods}

\section{Study population}

In this single center retrospective cohort study, all patients who received a TEE examination between 1 Jan-
Fig. 1 Representative images displaying a transesophageal short axis view of the descending thoracic aorta (panel a) without (left side) and with (right side) aortic plaque, a transesophageal four-chamber view (panel b) with normal (left side) and severely calcified (right side) mitral valve, and a transesophageal short axis view of the aortic valve (panel c) with normal (left side) and severely calcified (right side) morphology
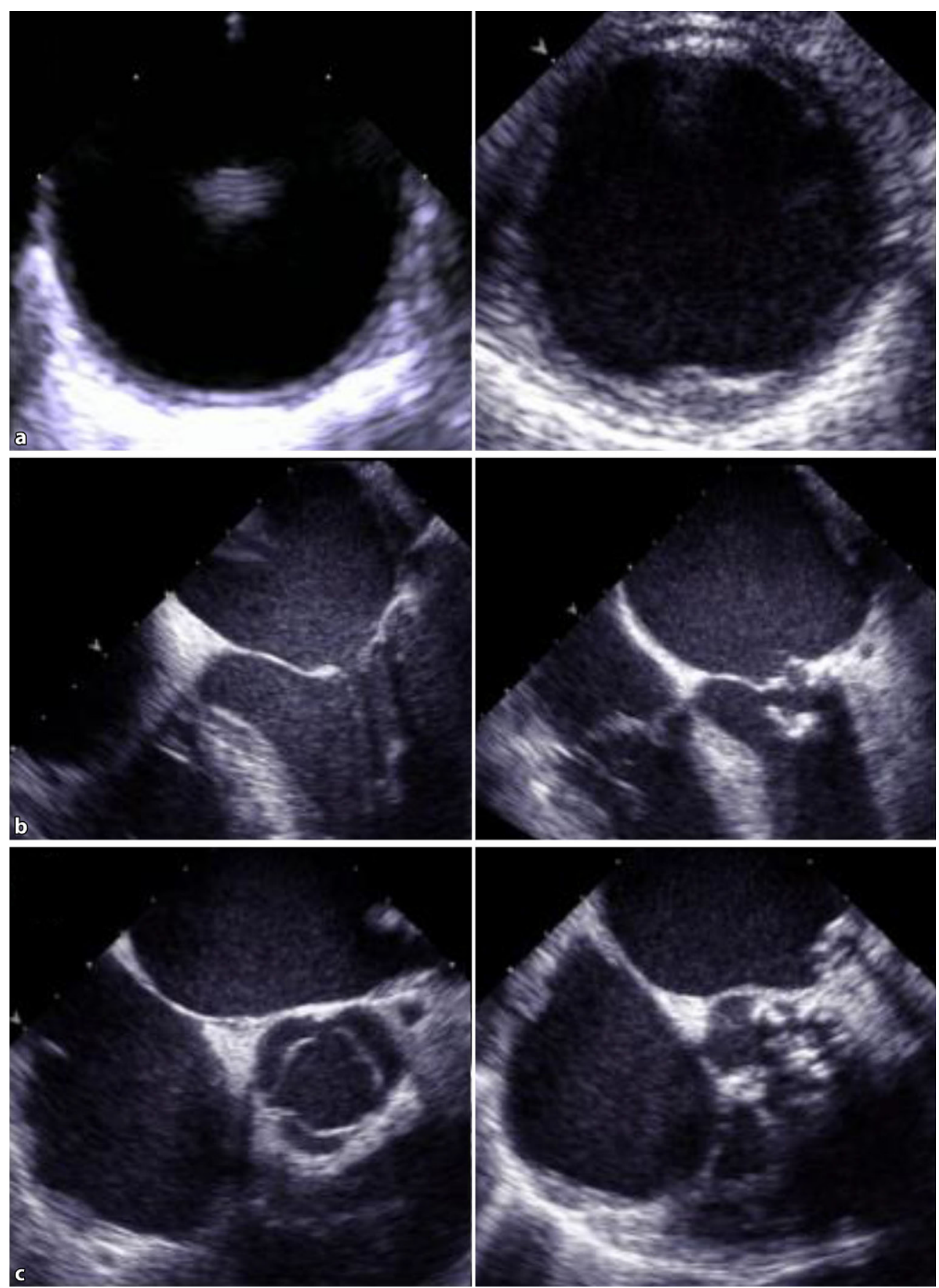
Table 1 Patient characteristics, echocardiographic and invasive data $(n=242)$

\begin{tabular}{|c|c|c|c|c|}
\hline & Total & No CAD & CAD & $p$-value \\
\hline \multicolumn{5}{|l|}{ Patient characteristics } \\
\hline Number of patients, $n(\%)$ & $242(100)$ & $104(43)$ & $138(57)$ & - \\
\hline Age, mean years (SD) & $70( \pm 13)$ & $65( \pm 15)$ & $73( \pm 11)$ & $<0.001$ \\
\hline Male sex, $n(\%)$ & $147(61)$ & $61(59)$ & $86(62)$ & 0.565 \\
\hline \multicolumn{5}{|l|}{ Cardiovascular risk factors } \\
\hline Arterial hypertension, $n(\%)$ & $183(76)$ & $67(64)$ & $116(84)$ & $<0.001$ \\
\hline Diabetes mellitus, $n(\%)$ & $64(26)$ & $19(18)$ & $45(33)$ & 0.012 \\
\hline Hyperlipidemia, $n(\%)$ & $105(43)$ & $24(23)$ & $81(59)$ & $<0.001$ \\
\hline Smoking, $n(\%)$ & $72(30)$ & $25(24)$ & $47(34)$ & 0.092 \\
\hline \multicolumn{5}{|l|}{ Medication } \\
\hline Anticoagulation, $n(\%)$ & $111(46)$ & $57(55)$ & $54(39)$ & 0.015 \\
\hline Antiplatelet therapy, $n(\%)$ & $124(51)$ & $30(29)$ & $94(68)$ & $<0.001$ \\
\hline ACE inhibitor, $n(\%)$ & $173(72)$ & $63(61)$ & $110(80)$ & 0.001 \\
\hline Beta blocker, $n(\%)$ & $163(67)$ & $63(61)$ & $100(73)$ & 0.051 \\
\hline Diuretics, $n(\%)$ & $170(70)$ & $67(64)$ & $103(75)$ & 0.086 \\
\hline \multicolumn{5}{|l|}{ Echocardiographic data } \\
\hline Plaque thoracic aorta, $n(\%)$ & $119(49)$ & $47(45)$ & $72(52)$ & 0.058 \\
\hline Plaque AscAo, $n(\%)$ & $33(14)$ & $9(9)$ & $24(17)$ & 0.05 \\
\hline Plaque AoArch, $n(\%)$ & 74 (31) & $29(28)$ & $45(33)$ & 0.335 \\
\hline Plaque DesAo, $n(\%)$ & $67(28)$ & $25(24)$ & $42(30)$ & 0.056 \\
\hline Aortic stenosis $\geq$ mild, $n(\%)$ & $87(36)$ & $24(23)$ & $63(46)$ & $<0.001$ \\
\hline MAC, $n(\%)$ & $88(36)$ & $24(23)$ & $64(46)$ & $<0.001$ \\
\hline \multicolumn{5}{|l|}{ Indication for TOE } \\
\hline Evaluation of valve disease, $n(\%)$ & $108(44.6)$ & $50(48.1)$ & $67(48.5)$ & 0.879 \\
\hline Exclusion of intracardiac thrombi, $n(\%)$ & $53(21.9)$ & $25(24)$ & $28(20.3)$ & 0.487 \\
\hline Endocarditis, $n(\%)$ & $35(14.5)$ & $13(12.5)$ & $22(15.9)$ & 0.453 \\
\hline Embolic stroke patient: Search for origin of emboly, $n$ (\%) & $20(8.3)$ & $5(4.8)$ & $14(10.1)$ & 0.128 \\
\hline Suspicion for intracardiac shunt lesion, $n(\%)$ & $13(5.4)$ & $9(8.7)$ & $4(2.9)$ & 0.05 \\
\hline Intracardiac tumor, $n(\%)$ & $4(1.7)$ & $2(1.9)$ & $2(1.4)$ & 0.776 \\
\hline \multicolumn{5}{|l|}{ Indication for CA } \\
\hline Preoperative CAD evaluation, $n(\%)$ & $145(59.9)$ & $69(66.3)$ & $76(55.1)$ & 0.071 \\
\hline Clinical suspicion for CAD, $n(\%)$ & $79(32.6)$ & $32(30.8)$ & $47(34.1)$ & 0.599 \\
\hline Acute coronary syndrome, $n(\%)$ & $16(6.6)$ & $2(1.9)$ & $14(10.1)$ & 0.011 \\
\hline
\end{tabular}

uary 2007 and 30 June 2016 and who had coronary angiography within 24 months before or after the TEE were evaluated. The ethics committee of the Medical University of Vienna (Nr. 1725/2016) approved the study in accordance with the Declaration of Helsinki.

In the searched period, 242 patients had TEE reports clearly describing the degree of aortic plaque, and the presence or absence of MAC and aortic stenosis (AS), and complete documentation of coronary angiography.

A systematic exploration of the centralized patient management system of Vienna (AKIM-AKH-Informationsmanagement) was performed to obtain information on medical diagnoses and daily medication.

\section{Echocardiography}

Data on echocardiographic examinations were retrospectively extracted from the hospital database. Examinations were performed with the following echo machines: Siemens Acuson (Siemens, Munich, Germany), Philips iE33 (Koninklijke Philips N.V. Amsterdam, Netherlands), Acuson Sequoja 512 (Siemens, Munich, Germany), and GE Vivid 7 (General Electric Healthcare, Chicago, IL, USA). At our institution the morphology of the aorta is visualized and described in every TEE examination.

The degree of calcification is reported as no plaque, minimal plaque, minimal to moderate plaque, moderate plaque, moderate to severe plaque, and severe plaque. The differentiation between the groups is made by visual quantification only. To minimize inter- 
observer variability and to reliably identify those patients with relevant aortic plaque, we evaluated two groups, one with minimal or no aortic plaque and one with more than minimal aortic plaque. Presence of MAC was diagnosed by visual assessment and defined as the presence of at least mild calcification of the mitral annulus. We evaluated two groups, one without MAC and one with at least mild MAC. Presence of aortic stenosis (AS) was diagnosed by visual assessment as well as by interpretation of continuous wave Doppler measurements. We evaluated two groups, one without AS and one with at least mild AS (Fig. 1).

\section{Coronary angiography}

We retrospectively extracted reports of the coronary angiograms. Presence of CAD was defined as any lesion in at least one of the coronary arteries. We further divided in the two groups significant and non-significant CAD, while significant obstructive CAD was defined as $\geq 70 \%$ stenosis of $\geq 1$ major branch.

\section{Statistical analysis}

Continuous variables are given as mean \pm standard deviation (SD). Categorical data are presented as absolute numbers and percentages. Comparison of categorical data was performed using Student's unpaired t-test analysis. A $P$-value of $<0.05$ was considered statistically significant. Positive and negative predictive values were calculated for the prediction of presence and absence of CAD. Univariate and multivariate logistic regression analyses were applied to examine the relationships between presence and absence of $\mathrm{CAD}$ and aortic plaque, MAC, and AS. The multivariate model was adjusted for established cardiovascular risk factors (arterial hypertension, diabetes mellitus, history of smoking, hyperlipidemia, and age) to control for potential confounding. The SPSS Version 24 (IBM SPSS, USA) was used for all analyses.

\section{Results}

\section{Patient characteristics}

At total of 242 patients fulfilled the inclusion criteria, $61 \%$ were male. Mean age was 70 (SD \pm 13 ) years. All patients received TEE and coronary angiography within 24 months, mean time between the 2 examinations was 99 days. Most common indications for TEE were preoperative/preinterventional evaluation of valve disease $(44.6 \%)$, exclusion of intracardiac thrombi (21.9\%), and suspicion for endocarditis (14.5\%). Indications for coronary angiography were preoperative angiography (59.9\%), clinical suspicion of CAD $(32.6 \%)$ and acute coronary syndrome $(6.6 \%)$.

Normal coronary arteries were present in 104 (43\%) of the patients. Significant CAD was present in 98
(41\%) of the patients. Left main stenosis was present in 7 patients, 6 of those had concomitant CAD with at least 1 other stenosis $\geq 70 \%, 1$ patient had a $90 \%$ isolated left main stenosis. In the overall group 119 $(49 \%)$ patients had plaque in at least 1 part of the thoracic aorta, $87(36 \%)$ had at least mild AS, and 88 $(36 \%)$ had at least mild MAC. Plaque in the ascending aorta was found in $14 \%$, in the aortic arch in $31 \%$, and in the descending aorta in $28 \%$.

\section{Patients with coronary artery disease}

A total of 138 (57\%) patients had CAD. When compared to those without CAD, they were older (73 years vs. 65 years, $p<0.001)$, and arterial hypertension $(84 \%$ vs. $64 \%, p<0.001$ ), diabetes mellitus (33\% vs. $18 \%$, $p=0.012$ ), and hyperlipidemia (59\% vs. $23 \%, p<0.001$ ) were more common. While there was no significant difference in presence of plaque in the aorta, MAC $(46 \%$ vs. $23 \%, p<0.001)$ and at least mild AS (46\% vs. $23 \%, p<0.001$ ) were more frequent (Table 1 ).

\section{Prediction of the presence or absence of significant coronary artery disease}

In univariate analysis, plaque in the ascending (odds ratio $[\mathrm{OR}] 2.23,95 \%$ confidence interval [CI] 1.06-4.7, $p=0.034$ ) and descending aorta (OR 2.05, 95\% CI 1.03-4.07, $p=0.041$ ) as well as in the aortic arch (OR 2.84, 95\% CI 1.29-6.28, $p=0.01$ ), at least mild AS (OR 1.79, 95\% CI 1.05-3.06, $p=0.033$ ), at least mild MAC (OR 2.52, 95\% CI 1.47-4.33, $p=0.001$ ), and any two of the previous in combination (OR 2.3, 95\% CI 1.29-4.11, $p=0.005$ ) were significant predictors of relevant $\mathrm{CAD}$ (at least one $70 \%$ stenosis), data not shown.

Table 2 Association of age, sex, cardiovascular risk factors (diabetes, hypertension, hyperlipidemia, smoking), and presence of aortic plaque, mitral annular calcification (MAC), and aortic stenosis (AS) with the presence of significant and with any coronary artery disease (CAD)

\begin{tabular}{|l|l|l|l|}
\hline $\begin{array}{l}\text { Variable } \\
\text { Multivariate analysis }\end{array}$ & OR & $95 \% \mathrm{Cl}$ & $p$-value \\
$\begin{array}{l}\text { Presence of significant CAD } \\
\text { Plaque in the ascending aorta }\end{array}$ & 2.51 & $1.18-5.32$ & $\mathbf{0 . 0 1 7}$ \\
\hline $\begin{array}{l}\text { Plaque in the aortic arch } \\
\text { Plaque in the descending aorta }\end{array}$ & 2.09 & $0.81-5.36$ & 0.127 \\
\hline $\begin{array}{l}\text { Plaque in at least one of the aortic } \\
\text { segments }\end{array}$ & 1.6 & $0.72-3.55$ & 0.244 \\
\hline At least mild MAC & 1.07 & $1.02-4.22$ & $\mathbf{0 . 0 4 5}$ \\
\hline At least mild AS & 1.51 & $1.01-3.36$ & $\mathbf{0 . 0 4 6}$ \\
\hline Presence of any CAD & $0.79-2.89$ & 0.21 \\
\hline At least mild AS & 2.53 & $1.23-5.21$ & $\mathbf{0 . 0 1 2}$ \\
\hline MAC & 1.86 & $0.97-3.59$ & 0.063 \\
\hline MAC and AS & 1.92 & $0.88-4.19$ & 0.102 \\
\hline Bold values indicate statistical significance with a & $p$-value $<0.05$ & \\
\hline
\end{tabular}


Table 3 Sensitivity, specificity, negative predictive value (NPV), positive predictive value (PPV), and accuracy of TEE signs of atherosclerosis predicting significant coronary artery disease (CAD)

\begin{tabular}{l|l|l|l|l|l|}
\hline \multicolumn{7}{l}{ Prediction of significant CAD by TEE signs of atherosclerosis } & \multicolumn{1}{l}{} \\
\hline Plaque in the ascending aorta & Sensitivity (\%) & Specificity (\%) & NPV (\%) & PPV (\%) & Accuracy (\%) \\
\hline Plaque in the aortic arch & $29(16-46)$ & $81(70-90)$ & $66(61-71)$ & $48(32-65)$ & $62(52-71)$ \\
\hline Plaque in the descending aorta & $74(60-86)$ & $47(36-59)$ & $74(63-83)$ & $47(41-54)$ & $58(49-67)$ \\
\hline Plaque in at least one of the aortic segments & $57(42-71)$ & $60(50-70)$ & $73(65-79)$ & $43(35-52)$ & $59(51-67)$ \\
\hline MAC & $72(61-82)$ & $48(40-57)$ & $75(66-82)$ & $45(40-51)$ & $57(50-64)$ \\
\hline AS & $49(39-60)$ & $72(64-79)$ & $68(63-72)$ & $55(46-63)$ & $63(56-69)$ \\
\hline None present & $44(34-55)$ & $69(61-77)$ & $65(60-69)$ & $49(41-58)$ & $59(53-65)$ \\
\hline All present & $87(78-93)$ & $34(26-43)$ & $80(69-87)$ & $46(43-50)$ & $55(48-62)$ \\
\hline AS aortic stenosis, MAC mitral annular calcification & $16(10-26)$ & $88(82-93)$ & $62(59-64)$ & $48(33-64)$ & $60(53-66)$ \\
\hline
\end{tabular}

In multivariate regression analysis, plaque in the ascending aorta (OR 2.51, 95\%CI 1.18-5.32, $p=0.017$ ), plaque in at least one of the thoracic aortic segments (OR 2.07, 95\%CI 1.02-4.22, $p=0.045$ ), and the presence of MAC (OR 1.84, 95\%CI 1.01-3.36, $p=0.046$ ) were predictors of significant CAD. In the presence of MAC, AS, and aortic plaque at the same time, there was no association with the presence of significant CAD (OR 1.15, 95\%CI 0.52-2.57, $p=0.732$ ). For data on regression analysis regarding the presence of significant CAD see Table 2.

Negative predictive value (NPV) for the presence of significant CAD was $80 \%$ if there was neither MAC, nor AS, nor aortic plaque (Table 3).

\section{Prediction of the presence or absence of normal coronary arteries}

In univariate analysis, none of the aortic plaque regions for themselves reliably detected the presence or absence of CAD. In multivariate regression analysis, the isolated finding aortic stenosis (OR 2.53, 95\% CI 1.23-5.21, $p=0.012$ ) was a significant predictor for the absence of normal coronary arteries, see Table 2 .

The PPV for the presence of any CAD was $73 \%$ for MAC, $72 \%$ for AS, and $76 \%$ if both AS and MAC were present, data not shown.

\section{Discussion}

In times of modern imaging systems, it is possible to evaluate the exact morphology of the mitral and aortic valves as well as the wall of the thoracic aorta via TEE in almost every patient. When analyzing these findings of generalized atherosclerosis, it is important to draw the correct conclusions. With our data, we could show that while the absence of MAC, AS, and aortic plaque can predict the absence of significant $\mathrm{CAD}$, and the presence of AS can predict the absence of normal coronary arteries, the presence of severe multifocal generalized atherosclerosis is more a sign of age-related degeneration than a reliable predictor of CAD.
Assessment of plaque in the thoracic aorta as well as of MAC and AS is part of every routine TEE examination [25]. Their presence and their predictive power regarding CAD have been analyzed in numerous studies in the past decades $[5-10,12,15,16]$. When interpreting previous studies, it is of great importance to differentiate those studies which analyzed TTE images from those which analyzed TEE images. There are two biases that must be considered: one is that determining low-grade MAC or AVS can be challenging by TTE but is easy and reliable in TEE due to superior image quality. The other is that most of the studies cited are retrospective data analyses, which leads to a significant selection bias between one group which received TTE and coronary angiography (bias towards acute coronary syndromes) and the other which received TEE and coronary angiography (bias towards valvular heart disease) in close time intervals. In addition, there has been a shift in technology in recent years. The earlier studies did not only use outdated ultrasound machines for diagnosis but also methods such as M-mode which do not compare to today's possibilities with direct visualization of calcifications in TEE imaging.

In the following, two important clinical questions are addressed. One is the diagnostic performance of signs of general atherosclerosis detected in high-end TEE imaging in the exclusion of CAD. The other is the prediction of significant CAD.

\section{Predicting significant coronary artery disease}

The diagnosis of significant CAD has a direct impact on the prognosis of the patient. In our data, plaque in the ascending aorta and plaque detected in at least one segment of the thoracic aorta were strong predictors of significant CAD in multivariate regression analysis. If there were no aortic plaques, NPV for the presence of significant $\mathrm{CAD}$ was $75 \%$. MAC and the presence of two out of the three (MAC, AS, aortic plaque) also predicted significant CAD and AS as a single parameter as well as the presence of all three (MAC, AS, aortic plaque) did not predict significant CAD. If there 
was neither MAC, nor AS, or aortic plaque, NPV for significant $\mathrm{CAD}$ was $80 \%$.

Since abnormal flow patterns in congenitally altered aortic valves such as bicuspid aortic valves can cause premature aortic stenosis, there is an overlap between those who develop premature degenerative AS and those who develop AS due to congenitally altered valves. Therefore, AS can be a misleading sign for generalized atherosclerosis. If bicuspid aortic valves can be excluded, AVS was a predictor for CAD in a previous study [8]. This differentiation was impossible in our analysis due to the retrospective nature of the study and may explain why AS was not a significant predictor of the presence of significant CAD in our data.

The presence of MAC, AS, and aortic plaque implies severe generalized atherosclerosis, which can develop due to severe cardiovascular risk profiles or due to advanced age. Interestingly, this pattern is not necessarily associated with the presence of severe CAD. Of the 32 patients with calcifications in all 3 regions, 16 (50\%) had significant CAD, and 8 (25\%) had normal coronary arteries. Thus, degenerative findings in TEE examination must be interpreted carefully regarding the presence of CAD.

\section{Excluding coronary artery disease}

Negative predictive value of coronary computed tomography angiography (CCTA) for the presence of CAD reaches $97-99 \%$ [26-29] but CCTA is less reliable in patients with coronary artery calcification. Diagnostic dilemmas are calcifications detected by CCTA, which later turn out to be non-significant in angiography. In these cases, CCTA exposed the patient to unnecessary contrast agent and radiation. Therefore, the most important differentiation by non-invasive or minimally invasive imaging regarding $\mathrm{CAD}$ must be the wise selection of those patients who most likely do not have $\mathrm{CAD}$ to steer decision making regarding CCTA versus coronary angiography. In many patients where CAD needs to be excluded, TEE examination has been performed before, e.g. in patients with endocarditis, mitral valve pathologies requiring surgery, and in patients with atrial fibrillation where there is clinical suspicion for CAD.

The current guidelines suggest considering the presence of decreased left ventricular function and regional wall motion abnormalities to decide about further diagnostic tests [30]. Secondary echocardiographic findings such as calcification and sclerosis of valves and vessels are not used for determination of pretest probability for CAD. This is because of conflicting data regarding the association of general degenerative findings with the presence of CAD.

In our data, there was no significant association between the presence of aortic plaque with the presence or absence of CAD. This can be attributed to the fact that our patient collective had a high prevalence of cardiovascular risk factors and in the group with normal coronary arteries, aortic plaque could be found in as many as $28 \%$ in the aortic arch and in $24 \%$ in the descending aorta; however, the isolated finding of at least mild AS was a significant predictor for CAD in multivariate regression analysis. The PPV for the presence of CAD was $73 \%$ for MAC, $72 \%$ for AS, and $76 \%$ if both AS and MAC were present. If there was MAC, AS, or plaque in at least one segment of the aorta, sensitivity for the presence of any CAD was $83 \%$.

Previous data have shown differing results, partly with significantly better results regarding the prognostic value of echo findings; however, the study designs differed tremendously. Some studies considered a coronary artery stenosis of at least $50 \%$ to be significant $[18,19,21]$, another study was retrospective and with few patients [20].

In our study, there was an independent association of MAC with the presence of significant CAD in multivariate regression analysis. This is in contrast to previous data, e.g. by Bhatt $\mathrm{H}$ et al. [17]. This group retrospectively looked at patients who had received TTE and coronary angiography within 3 years. It can be assumed that this study included a different patient population than ours, e.g. patients with acute coronary syndrome. In our cohort, only $6.6 \%$ of patients presented with ACS. The majority were evaluated for elective valvular heart surgery/intervention. It can be concluded that prospective data are needed to clarify the relevance of MAC and other signs of atherosclerosis in the prediction of CAD.

These data can be understood as hypothesis generating: If there are no signs of atherosclerosis in the TEE examination performed for the preinterventional or preoperative evaluation of valvular heart disease, CCTA might be sufficient for exclusion of CAD independent of the age of the patient.

\section{Limitations}

This study has limitations. The data collection was retrospective; the reported values were exported from the hospital database. A cohort which has received both coronary angiography and TEE examination at a tertiary medical center within 24 months cannot be compared to the normal population; however, despite the high prevalence of generalized atherosclerosis in our patient group, there were nevertheless significant differences between the analyzed groups. A prospective study with careful inclusion of participants is needed to clarify this bias, which has certainly affected the previously published retrospective studies as well.

\section{Conclusion}

With modern ultrasound systems, the presence of MAC, AS, and aortic plaque can be examined reliably in almost all TEE examinations. With an NPV of 
$80 \%$, their absence makes the presence of significant CAD unlikely. If at least mild AS is present, normal coronary arteries are improbable.

Funding Open access funding provided by Medical University of Vienna.

Conflict of interest M. Schneider, H. Heidari, H. Ran, C. Roth, C. Hengstenberg, T. Binder, and G. Goliasch declare that they have no competing interests.

Open Access This article is licensed under a Creative Commons Attribution 4.0 International License, which permits use, sharing, adaptation, distribution and reproduction in any medium or format, as long as you give appropriate credit to the original author(s) and the source, provide a link to the Creative Commons licence, and indicate if changes were made. The images or other third party material in this article are included in the article's Creative Commons licence, unless indicated otherwise in a credit line to the material. If material is not included in the article's Creative Commons licence and your intended use is not permitted by statutory regulation or exceeds the permitted use, you will need to obtain permission directly from the copyright holder. To view a copy of this licence, visit http://creativecommons.org/licenses/by/4.0/.

\section{References}

1. Boon A, Cheriex E, Lodder J, Kessels F. Cardiac valve calcification: characteristics of patients with calcification of the mitral annulus or aortic valve. Heart. 1997;78(5):472-4.

2. Allison MA, Cheung P, Criqui MH, Langer RD, Wright CM. Mitral and aortic annular calcification are highly associated with systemic calcified atherosclerosis. Circulation. 2006;113(6):861-6.

3. Hamirani YS, Nasir K, Blumenthal RS, Takasu J, Shavelle D, Kronmal R, et al. Relation of mitral annular calcium and coronary calcium (from the multi-ethnic study of atherosclerosis [MESA]). Am J Cardiol. 2011;107(9):1291-4.

4. Committee CS. A randomised, blinded, trial of clopidogrel versus aspirin in patients at risk of ischaemic events (CAPRIE). CAPRIE steering committee. Lancet. 1996;348(9038):1329-39.

5. Fox CS, Vasan RS, Parise H, Levy D, O’Donnell CJ, D'Agostino RB, et al. Mitral annular calcification predicts cardiovascular morbidity and mortality: the Framingham heart study. Circulation. 2003;107(11):1492-6.

6. Kohsaka S, Jin Z, Rundek T, Boden-Albala B, Homma S, Sacco RL, et al. Impact of mitral annular calcification on cardiovascular events in a multiethnic community: the northern Manhattan study. JACC Cardiovasc Imaging. 2008;1(5):617-23.

7. Jeon DS, Atar S, Brasch AV, Luo H, Mirocha J, Naqvi TZ, et al. Association of mitral annulus calcification, aortic valve sclerosis and aortic root calcification with abnormal myocardial perfusion single photon emission tomography in subjects age $<$ or $=65$ years old. J Am Coll Cardiol. 2001;38(7):1988-93.

8. Conte L, Rossi A, Cicoira M, Bonapace S, Amado EA, Golia G, et al. Aortic valve sclerosis: a marker of significant obstructive coronary artery disease in patients with chest pain? JAm Soc Echocardiogr. 2007;20(6):703-8.

9. AtarS, JeonDS, Luo H, SiegelRJ. Mitral annular calcification: a marker of severe coronary artery disease in patients under 65 years old. Heart. 2003;89(2):161-4.

10. Lange DC, Glidden D, Secemsky EA, Ordovas K, Deeks SG, Martin JN, et al. Mitral annular and coronary artery calcification are associated with mortality in HIV-infected individuals. PLoSOne. 2015;10(7):e130592.

11. Thanassoulis G, Massaro JM, Cury R, Manders E, Benjamin EJ, Vasan RS, et al. Associations of long-term and early adult atherosclerosis riskfactors with aortic and mitral valve calcium. J Am Coll Cardiol. 2010;55(22):2491-8.

12. Acartürk E, Bozkurt A, Cayli M, Demir M. Mitral annular calcification and aortic valve calcification may help in predicting significant coronary artery disease. Angiology. 2003;54(5):561-7.

13. Kamenský G, Lisy L, Polak E, Piknova E, Plevova N. Mitral annular calcifications and aortic plaques as predictors of increased cardiovascular mortality. J Cardiol. 2001;37(1):21-6.

14. Rao AK, Djamali A, Korcarz CE, Aeschlimann SE, Wolff MR, Stein JH. Mitral annular calcification is associated with reduced left ventricular function and inflammation in patients with chronic kidney disease. J Am Soc Echocardiogr. 2008;21(6):747-50.

15. Adler Y, Vaturi M, Fink N, Tanne D, Shapira Y, Weisenberg D, et al. Association between mitral annulus calcification and aortic atheroma: a prospective transesophageal echocardiographic study. Atherosclerosis. 2000;152(2):451-6.

16. Koulaouzidis G, Nicoll R, MacArthur T, Jenkins PJ, Henein MY. Coronary artery calcification correlates with the presence and severity of valve calcification. IntJCardiol. 2013;168(6):5263-6.

17. Bhatt H, Sanghani D, Julliard K, Fernaine G. Is mitral annular calcification associated with atherosclerotic risk factors and severity and complexity of coronary artery disease? Angiology. 2015;66(7):659-66.

18. Frogoudaki A, Barbetseas J, Aggeli C, Panagiotakos D, Lambrou S, Pitsavos C, et al. Thoracic aorta atherosclerosis burden index predicts coronary artery disease in patients undergoing transesophageal echocardiography. Atherosclerosis. 2008;197(1):232-6.

19. Kim HY, Kim CJ, Rho TH, Youn HJ, Jin SW, Rhim HY, et al. Transesophageal echocardiographic detection of thoracic aortic plaque could noninvasively predict significant obstructive coronary artery disease. Korean J Intern Med. 1999;14(2):20-6.

20. Tribouilloy C, Shen WF, Peltier M, Lesbre JP. Noninvasive prediction of coronary artery disease by transesophageal echocardiographic detection of thoracic aortic plaque in valvular heart disease. Am J Cardiol. 1994;74(3):258-60.

21. Leskinen Y, Groundstroem K, Virtanen V, Lehtimäki T, Huhtala H, Saha H. Prediction of coronary artery disease by transesophageal echocardiographic detection of thoracic aortic plaque in patients with chronic kidney disease. Nephron Clin Pract. 2006;103(4):c157-61.

22. GuX, HeY, Li Z, Kontos MC, Paulsen WH, Arrowood JA, et al. Relation between the incidence, location, and extent of thoracicaortic atherosclerosis detected by transesophageal echocardiography and the extent of coronary artery disease by angiography. Am J Cardiol. 2011;107(2):175-8.

23. Izumi C, Takahashi S, Miyake M, Sakamoto J, Hanazawa K, Yoshitani K, et al. Impact of aortic plaque morphology on survival rate and incidence of a subsequent embolic event-long-term follow-up data. Circ J. 2010;74(10):2152-7.

24. Amarenco P, Cohen A, Tzourio C, Bertrand B, Hommel M, Besson G, et al. Atherosclerotic disease of the aortic arch and the risk of ischemic stroke. N Engl J Med. 1994;331(22):1474-9.

25. Hahn RT, Abraham T, Adams MS, Bruce CJ, Glas KE, Lang RM, et al. Guidelines for performing a comprehensive transesophageal echocardiographic examination: 
recommendations from the American society of echocardiography and the society of cardiovascular anesthesiologists. JAm Soc Echocardiogr. 2013;26(9):921-64.

26. Budoff MJ, Dowe D, Jollis JG, Gitter M, Sutherland J, Halamert E, et al. Diagnostic performance of 64-multidetector row coronary computed tomographic angiography for evaluation of coronary artery stenosis in individuals without known coronary artery disease: results from the prospective multicenter ACCURACY (assessment by coronary computed tomographic angiography of individuals undergoing invasive coronary angiography) trial. J Am Coll Cardiol. 2008;52(21):1724-32.

27. Meijboom WB, Meijs MF, Schuijf JD, Cramer MJ, Mollet NR, van Mieghem CA, et al. Diagnostic accuracy of 64-slice computed tomography coronary angiography: a prospective, multicenter, multivendor study. J Am Coll Cardiol. 2008;52(25):2135-44.
28. Paech DC, Weston AR. A systematic review of the clinical effectiveness of 64-slice or higher computed tomography angiography as an alternative to invasive coronary angiography in the investigation of suspected coronary artery disease. BMCCardiovasc Disord. 2011;11:32.

29. Li M, Du XM, Jin ZT, Peng ZH, Ding J, Li L. The diagnostic performance of coronary artery angiography with 64-MSCT and post 64-MSCT: systematic review and meta-analysis. PLoSOne. 2014;9(1):e84937.

30. Montalescot G, Sechtem U, Achenbach S, Andreotti F, Arden C, Budaj A, et al. ESC guidelines on the management of stable coronary artery disease: the task force on the management of stable coronary artery disease of the European society of cardiology. Eur Heart J. 2013;34(38):2949-3003.

Publisher's Note Springer Nature remains neutral with regard to jurisdictional claims in published maps and institutional affiliations. 